\title{
Compensation for acoustic heterogeneities in photoacoustic computed tomography using a variable temporal data truncation reconstruction method
}

Joemini Poudel, Thomas P. Matthews, Mark A. Anastasio, Lihong V. Wang

Joemini Poudel, Thomas P. Matthews, Mark A. Anastasio, Lihong V. Wang, "Compensation for acoustic heterogeneities in photoacoustic computed tomography using a variable temporal data truncation reconstruction method," Proc. SPIE 9708, Photons Plus Ultrasound: Imaging and Sensing 2016, 97083W (15 March 2016); doi: 10.1117/12.2213416

SPIE. Event: SPIE BiOS, 2016, San Francisco, California, United States 


\title{
Compensation for acoustic heterogeneities in photoacoustic computed tomography using a variable temporal data truncation reconstruction method
}

\author{
Joemini Poudel ${ }^{\mathrm{a}}$, Thomas P. Matthews ${ }^{\mathrm{a}}$, Mark A. Anastasio ${ }^{\mathrm{a}}$, and Lihong V. Wang

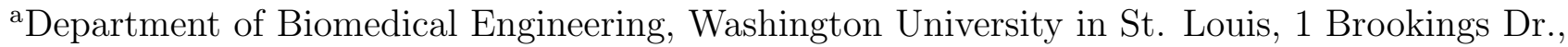 \\ St. Louis, MO, USA 63130
}

\begin{abstract}
Photoacoustic computed tomography (PACT) is an emerging computed imaging modality that exploits optical contrast and ultrasonic detection principles to form images of the absorbed optical energy density within tissue. If the object possesses spatially variant acoustic properties that are unaccounted for by the reconstruction algorithm, the estimated image can contain distortions. While reconstruction algorithms have recently been developed for compensating for this effect, they generally require the object's acoustic properties to be known a priori. To circumvent the need for detailed information regarding an object's acoustic properties, we have previously proposed a half-time reconstruction method for PACT. A half-time reconstruction method estimates the PACT image from a data set that has been temporally truncated to exclude the data components that have been strongly aberrated. In this approach, the degree of temporal truncation is the same for all measurements. However, this strategy can be improved upon when the approximate sizes and locations of strongly heterogeneous structures, such as gas voids or bones, are known. In this work, we investigate PACT reconstruction algorithms that are based on a variable temporal data truncation (VTDT) approach that represents a generalization of the half-time reconstruction approach. In the VTDT approach, the degree of temporal truncation for each measurement is determined by the distance between the corresponding transducer location and the nearest known bone or gas void location. Computer simulation studies are employed to demonstrate the feasibility and effectiveness of the approach.
\end{abstract}

Keywords: photoacoustic computed tomography, image reconstruction

\section{INTRODUCTION}

Photoacoustic computed tomography (PACT) is an emerging imaging modality with a wide range of applications. ${ }^{1-4}$ PACT exploits the high optical contrast of blood-rich structures and the high spatial resolution of ultrasound detection. In PACT, a short laser pulse is used to irradiate a biological sample, upon which optical absorption by tissues results in emission of pressure waves through the thermoacoustic effect. These pressure waves are subsequently detected by broadband ultrasound detectors. The image reconstruction problem in PACT is to estimate the absorbed optical energy density from the measured photoacoustic signals. Such an image may be of great importance as there exists a strong correlation between electromagnetic absorption and the pathological condition of tissue. ${ }^{5,6}$

Most PACT reconstruction algorithms assume an acoustically homogeneous medium. This can result in strong artifacts in the reconstructed images when the spatially variant acoustic properties of the medium are not taken into account. ${ }^{7,8}$ However, in practice, it is challenging to estimate these properties accurately. To circumvent the need for detailed information regarding an object's acoustic properties as well as suppress artifacts due to acoustic heterogeneity, we have previously proposed a half-time reconstruction method ${ }^{9}$ for PACT. The half-time reconstruction approach exploits data redundancies to uniquely and stably reconstruct images from measurement data truncated at a constant delay time. Although the half-time reconstruction approach reduces

Further author information: (Send correspondence to Mark A. Anastasio, Lihong V. Wang)

Mark A. Anastasio : E-mail: anastasio@wustl.edu

Lihong V. Wang : E-mail: lhwang@seas.wustl.edu

Photons Plus Ultrasound: Imaging and Sensing 2016, edited by Alexander A. Oraevsky, Lihong V. Wang Proc. of SPIE Vol. 9708, 97083W - (C) 2016 SPIE · CCC code: 1605-7422/16/\$18 - doi: 10.1117/12.2213416 
the acoustic heterogeneity-induced artifacts as compared to the full-time approach, ${ }^{9}$ significant distortions can remain. Moreover, half-time reconstruction algorithms do not employ any a priori information about the acoustic heterogeneities to guide the reconstruction algorithm.

In this work, we propose and investigate a PACT reconstruction algorithm that is based on a variable temporal data truncation (VTDT) approach. This algorithm represents a generalization of the half-time-based reconstruction algorithm and employs a priori information about the acoustic heterogeneities to guide the reconstruction algorithm. The degree of temporal truncation in the VTDT-based algorithm is dependent on the location of the acoustically heterogeneous region relative to the transducer positions. Due to the adaptive nature of the temporal truncation, artifacts arising from the acoustic heterogeneities are more effectively suppressed in the images reconstructed by use of the VTDT-based algorithm, as opposed to images reconstructed by use of the half-time-based algorithm.

\section{BACKGROUND}

In this section, we will provide an overview of the imaging model and the backprojection (BP) reconstruction algorithm. The temporal truncation strategies developed in subsequent sections will be applied to the BP reconstruction algorithm.

\subsection{Imaging Model}

Consider the case where an electromagnetic pulse with time dependence $\delta(t)$ is employed to irradiate tissue. The thermoacoustic effect will produce a pressure wave that will be measured by the wide band ultrasonic transducers that are located on the surface of a spherical measurement aperture $\partial \Omega$ that encloses the sample. In the 2 -D case, the measurement aperture $\partial \Omega$ corresponds to a circle. Assuming that the medium is acoustically homogeneous with spatially variant optical absorption properties, a mathematical relationship between the measured pressure data $g\left(\mathbf{r}_{\mathbf{0}}, t\right)$ at $\mathbf{r}_{\mathbf{0}} \in \partial \Omega$ and initial thermoacoustic pressure $p_{0}(\mathbf{r})$ within the object is given by ${ }^{9}$

$$
g\left(\mathbf{r}_{\mathbf{0}}, \bar{t}\right)=\int d^{3} \mathbf{r} p_{0}(\mathbf{r}) \frac{d}{d \bar{t}}\left(\frac{\delta\left(\bar{t}-\left|\mathbf{r}_{\mathbf{0}}-\mathbf{r}\right|\right)}{4 \pi\left|\mathbf{r}_{\mathbf{0}}-\mathbf{r}\right|}\right),
$$

where $c_{0}$ is the constant speed of sound and $\bar{t} \equiv c_{0} t$.

In practice, the detected pressure wavefield is discretized temporally and spatially at specific transducer locations. Let $\mathbf{g} \in \mathbb{R}^{N_{r} L}$ denote the discretized pressure signal, where $[\mathbf{g}]_{k N_{r}+l}=g\left(\mathbf{r}_{\mathbf{0}}{ }^{k}, l \Delta^{t}\right)$ such that $k=$ $0,1,2, \ldots, N_{r}-1$ and $l=0,1,2, \ldots, L-1$. Here, $L$ is the total number of temporal samples , $\Delta^{t}$ is the temporal sampling interval, and the vectors $\mathbf{r}_{\mathbf{0}}{ }^{k}, k=0,1,2, \ldots, N_{r}-1$, represent the $N_{r}$ receiver locations on the aperture $\partial \Omega$.

\subsection{Backprojection Algorithm}

Let us assume the object $\mathbf{p}_{\mathbf{0}}$ is represented on a 2-D Cartesian grid with $N=N_{1} N_{2}$ grid points, where $N_{i}$ denote the number of grid points along the $i^{t h}$ direction. Hence, the estimated initial pressure distribution, $\hat{\mathbf{p}_{\mathbf{0}}} \in \mathbb{R}^{N}$, obtained by the use of the $\mathrm{BP}$ algorithm is given by ${ }^{10}$

$$
\left[\hat{\mathbf{p}_{\mathbf{0}}}\right]_{m}=\sum_{k=0}^{N_{r}-1} \Delta \Omega_{k} B\left(\mathbf{g}, \frac{\left|\mathbf{r}_{\mathbf{0}}{ }^{k}-\mathbf{r}_{\mathbf{m}}\right|}{c_{0} \Delta^{t}}, k\right) / \sum_{k=0}^{N_{r}-1} \Delta \Omega_{k},
$$

where $\left[\hat{\mathbf{p}_{\mathbf{0}}}\right]_{m}$ is the $m^{\text {th }}$ element of $\hat{\mathbf{p}_{\mathbf{0}}}$ corresponding to grid position $\mathbf{r}_{\mathbf{m}}$ and $\Delta \Omega_{k}$ is computed by

$$
\Delta \Omega_{k}=\frac{\Delta S_{k}}{\left|\mathbf{r}_{\mathbf{m}}-\mathbf{r}_{\mathbf{0}}\right|^{2}}\left[\mathbf{n}_{0 k}^{S} \cdot \frac{\mathbf{r}_{\mathbf{m}}-\mathbf{r}_{\mathbf{0}}{ }^{k}}{\left|\mathbf{r}_{\mathbf{m}}-\mathbf{r}_{\mathbf{0}}{ }^{k}\right|}\right] .
$$

In Eqn. (3), $\Delta S_{k}$ represents the surface area occupied by the transducer at position $\mathbf{r}_{\mathbf{0}}{ }^{k}$ and $\mathbf{n}_{0 k}^{S}$ is the normal of the measurement surface at $\mathbf{r}_{\mathbf{0}}{ }^{k}$ that points towards the photoacoustic source. In addition, the function $B$ in Eqn. (2) is a linear interpolation function which is defined as

$$
B\left(\mathbf{g}, t_{v a l}, k\right)=\left(t_{v a l}-t_{i n t}\right)[\mathbf{g}]_{k L+\left(t_{i n t}+1\right)}+\left(t_{i n t}+1-t_{v a l}\right)[\mathbf{g}]_{k L+t_{i n t}},
$$

where $t_{\text {int }}=\left\lfloor t_{\text {val }}\right\rfloor$. 


\section{DATA TRUNCATION STRATEGIES}

In the preceding section, we discussed the full-time BP algorithm. In the full-time approach, the images are reconstructed from untruncated measurement data. Here, untruncated data refers to the data acquired at all time points such that pressure waves originating from any point on $\Omega$ reaches all the receivers on $d \Omega$. It has been shown that images reconstructed using full-time data can contain significant distortions when acoustic variations in the object are not accurately modeled. ${ }^{9}$ To address this problem, we proposed an image reconstruction algorithm based on a data truncation strategy known as the half-time method. ${ }^{9}$ In the half-time method, all transducer measurements are temporally truncated at a specific delay time $t_{\text {half }}$, where $t_{\text {half }}$ represents the time it takes for the pressure waves to propagate from a transducer on $d \Omega$ to the center of the scanning aperture.

We extend this idea further by adopting a data truncation strategy where the data for each transducer is truncated after a time delay that varies with transducer position. We refer to this as the VTDT approach. In this approach, the data for each transducer is truncated based on the distance between that transducer and the nearest acoustic heterogeneity. As a result, all data that has been distorted by traveling through an acoustic heterogeneity is eliminated from the image reconstruction process.

In both VTDT and half-time approaches, the temporal pressure data is truncated at specific time points such that not all data are backprojected. We can model this process by a truncation matrix $\mathbb{T} \in \mathbb{R}^{N_{r} L \times N_{r} L}$ that acts on $\mathbf{g}$ to produce a truncated pressure data vector $\mathbf{g}_{\text {Trunk }} \in \mathbb{R}^{N_{r} L}$ as

$$
\mathbf{g}_{\text {Trunk }}=\mathbb{T} \mathbf{g} .
$$

The construction of the truncation matrix differs between the half-time- and VTDT-based methods. The halftime truncation matrix, $\mathbb{T}_{H A L F}$, truncates all temporal data arriving after a specific delay time $t_{\text {half }}$. Here, $t_{\text {half }}$ is dependent on the radius of the circular measurement aperture as well as the assumed constant speed of sound value of the medium. The VTDT truncation matrix, $\mathbb{T}_{V T D T}$, however, truncates all temporal data that has been reflected off or transmitted through the acoustically heterogeneous region. Thus, $\mathbb{T}_{V T D T}$ is dependent on the distance between each of the transducers and the nearest known acoustic heterogeneity as well as the assumed constant speed of the sound of the medium.

\subsection{Backprojection algorithm based on temporal data truncation approaches}

In temporal truncation methods, the truncated pressure data vector is used by the BP algorithm to reconstruct $\hat{\mathbf{p}_{\mathbf{0}}}$. Thus, the reconstructed $\hat{\mathbf{p}_{\mathbf{0}}}$ is given by

$$
\left[\hat{\mathbf{p}_{\mathbf{0}}}\right]_{m}=\sum_{k=0}^{N_{r}-1} \Delta \Omega_{k} B\left(\mathbb{T} \mathbf{g}, \frac{\left|\mathbf{r}_{\mathbf{0}}{ }^{k}-\mathbf{r}_{\mathbf{m}}\right|}{c_{0} \Delta^{t}}, k\right) / \sum_{k=0}^{N_{r}-1} \Delta \Omega_{k} .
$$

In Eqn. (6), $\mathbb{T}$ can be equal to either $\mathbb{T}_{H A L F}$ or $\mathbb{T}_{V T D T}$ depending on whether we are using the half-time-based $\mathrm{BP}$ algorithm or the VTDT-based BP algorithm.

\section{COMPUTER SIMULATION STUDIES}

Computer-simulation studies were conducted to demonstrate the effectiveness and feasibility of the VTDT-based $\mathrm{BP}$ algorithm as compared to half-time-based BP algorithm.

\subsection{Methods}

A 2-D circular scanning geometry was employed to investigate the differences between VTDT- and half-timebased reconstruction algorithms. The scanning geometry utilized 512 transducers that were evenly distributed in a circle of diameter $50 \mathrm{~mm}$.

The three numerical phantoms used in the study to generate the forward data are shown in Figure 1. The acoustic heterogeneity used in the numerical simulation imitated an air void $\left(c_{0}=340 \frac{\mathrm{m}}{\mathrm{s}}\right.$ and $\left.\rho=1.2 \frac{\mathrm{kg}}{\mathrm{m}^{3}}\right)$ while the background medium consisted of water $\left(c_{0}=1500 \frac{\mathrm{m}}{\mathrm{s}}\right.$ and $\left.\rho=1000 \frac{\mathrm{kg}}{\mathrm{m}}\right)$. The initial pressure phantom consisted of two line absorbers placed perpendicular to one another. 

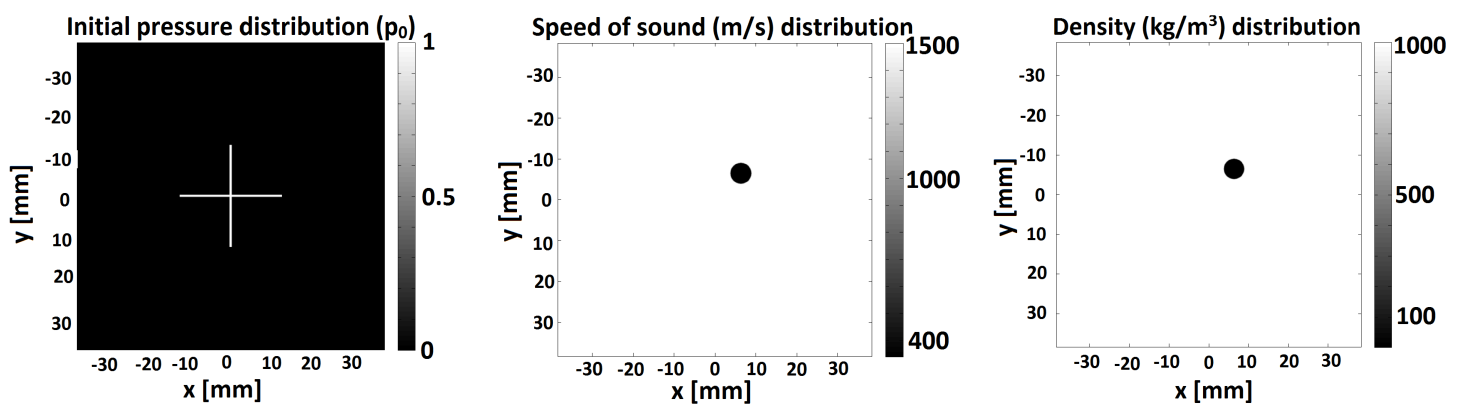

Figure 1: The initial pressure profile (left), speed of sound distribution (middle), and density distribution (right) used for computer simulation studies

Assuming ideal point-like transducers, the simulated pressure data corresponding to the numerical phantoms were calculated by use of the k-space pseudospectral method ${ }^{11-13}$ for the scanning geometry described. A 1536 $\times 1536$ grid with a pitch of $50 \mu \mathrm{m}$ was used to simulate the pressure data. A total of 5200 temporal samples were computed at each transducer location with a time step of $\Delta^{t}=6.25 \mathrm{~ns}$.

\subsection{Results}

For all the reconstruction methods, a constant speed of sound of $c_{0}=1500 \frac{\mathrm{m}}{\mathrm{s}}$ was assumed. For the VTDTbased BP algorithm, the truncation matrix $\mathbb{T}_{V T D T}$ was calculated based on the known location of the acoustically heterogeneous region and the assumed $c_{0}$. The truncation matrix was utilized to compute the truncated pressure data vector $\mathbf{g}_{\text {Trunk. }}$. Similarly, the truncation matrix and truncated pressure data vector were also computed for the half-time-based BP algorithm. Eqn. (6) was used to compute the reconstructed $\mathbf{p}_{\mathbf{0}}$ within a limited rectangular domain $(512 \times 512$ pixels $)$ with a grid size of $\Delta r=50 \mu \mathrm{m}$. The images reconstructed employing the half-time- and VTDT-based BP algorithms are shown in Fig 2. The VTDT approach truncates the reflected signals as well as those transmitted through the acoustically heterogeneous region. As a result, the artifacts due to acoustic heterogeneities are suppressed. In the half-time-based BP reconstructed image, artifacts due to
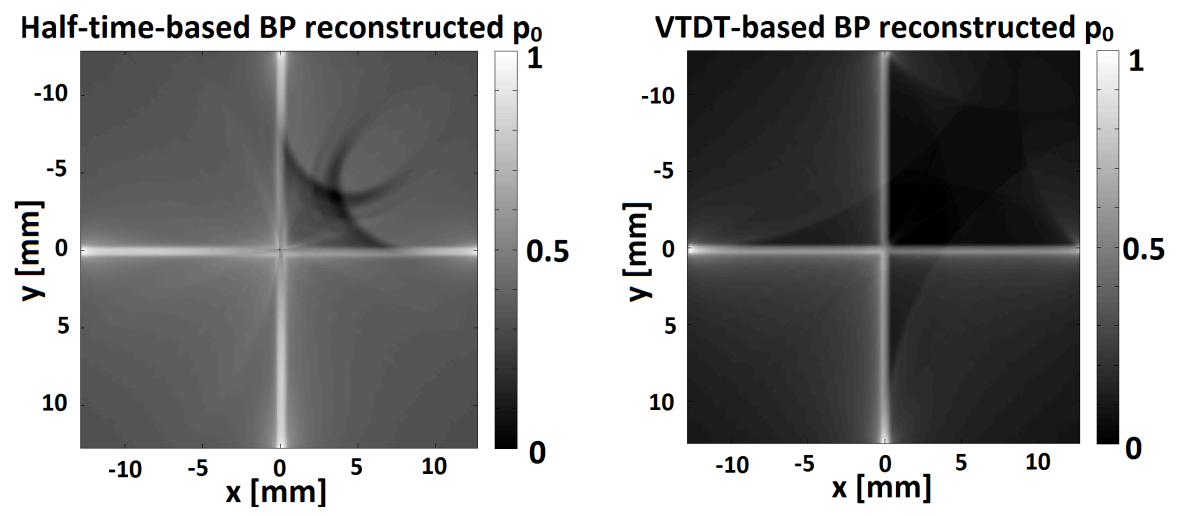

Figure 2: The normalized BP reconstructed images for half-time- (left) and VTDT-based (right) based approaches.

acoustic heterogeneities are visible in the upper right quadrant. We observe that the same artifacts are mitigated in the VTDT-based BP reconstructed image. Hence, we observe that the VTDT-based reconstruction algorithm utilizes a priori information about the object to mitigate artifacts due to acoustic heterogeneities. 


\section{CONCLUSION}

In this paper, we proposed and investigated a VTDT approach to PACT image reconstruction in acoustically heterogeneous media. The VTDT approach utilized the location of the acoustic heterogeneity to guide the temporal truncation of the PACT data. We observed that artifacts arising due to acoustically heterogeneous region was suppressed much more effectively in the VTDT-based BP reconstructed images as opposed half-timebased BP reconstructed images. This suggests that, for cases where a strong acoustic heterogeneity is present and its location is approximately known, we can adopt the VTDT-based reconstruction algorithm to improve reconstructed image quality.

However, if the size of the heterogeneity is large or if there are multiple heterogeneities, the VTDT approach may lead to excessive temporal truncation, resulting in poor reconstructed image quality. Thus, the VTDT approach may not be superior to half-time approaches for such cases.

\section{REFERENCES}

[1] R. Kruger, P. Liu, and C. Appledorn, "Photoacoustic ultrasound (paus) reconstruction tomography," Med. Phys. 22, pp. 605-1609, 1995.

[2] R. Kruger, D. Reinecke, and G. Kruger, "Thermoacoustic computed tomography- technical considerations," Med. Phys. 26, pp. 1832-1837, 1999.

[3] M. Xu and L. Wang, "Time-domain reconstruction for thermoacoustic tomography in a spherical geometry," IEEE Trans. Med. Imag. 21(7), pp. 814-822, 2002.

[4] M. Xu, Y. Xu, and L. Wang, "Time-domain reconstruction algorithms and numerical simulations for thermoacoustic tomography in various geometries," IEEE Trans. Biomed. Eng. 50(9), pp. 1086-1099, 2003.

[5] W. Jones, R. Jirtle, M. Rafal, and D. Schaeffer, "Microwave power absorption differences between normal and malignant tissue," Radiat. Oncol., Biol., Phys., 6, pp. 681-687, 1980.

[6] W. Cheong, R. Jirtle, M. Rafal, and D. Schaeffer, "Microwave power absorption differences between normal and malignant tissue," IEEE J. Quantum Elect. 26(12), pp. 1086-1099, 2003.

[7] X. Jin, C. Li, X. Pan, and L. Wang, "Effects of acoustic heterogeneities on transcranial brain imaging with microwave-induced thermoacoustic tomography," Med. Phys. 35(7), pp. 3205-3214, 2008.

[8] Y. Xu and L. Wang, "Effects of acoustic heterogeneity in breast thermoacoustic tomography," IEEE Trans. Ultrason., Ferroelectr., Freq. Contr. 50(9), pp. 1134-1146, 2003.

[9] M. Anastasio, J. Zhang, X. Pan, Y. Zou, G. Ku, and L. Wang, "Half-time reconstruction in thermoacoustic tomography," IEEE Trans. Med. Imag. 24(2), pp. 199-210, 2005.

[10] M. Xu and L. Wang, "Universal back-projection algorithm for photoacoustic computed tomography," Phys Rev.E 71, pp. 016706(1)-016706(7), 2005.

[11] T. Mast, L. Souriau, D. Liu, M. Tabei, A. Nachman, and R. Waag, "A k-space method for large-scale models of wave propagation in tissue," IEEE Ultrason., Ferroelectr., Freq. Control 48(2), pp. 341-354, 2001.

[12] M. Tabei, T. Mast, and R. Waag, "A k-space mthod for coupled first-order acoustic propogation equations," J. Acoust. Soc. 111(1), pp. 53-63, 2002.

[13] B. Treeby and B.T.Cox, "k-wave: Matlab toolbox for the simulation and reconstruction of photoacoustic wave-fields," J. Biomed. Opt. 15(2), p. 021314, 2010. 